\title{
Rehabilitation Exercises for the Gluteus Medius Muscle Segments: An Electromyography Study
}

\author{
Damien Moore, Tania Pizzari, Jodie McClelland, and Adam I. Semciw
}

\begin{abstract}
Context: Many different rehabilitation exercises have been recommended in the literature to target the gluteus medius (GMed) muscle based mainly on single-electrode, surface electromyography (EMG) measures. With the GMed consisting of 3 structurally and functionally independent segments, there is uncertainty on whether these exercises will target the individual segments effectively. Objective: To measure individual GMed segmental activity during 6 common, lower-limb rehabilitation exercises in healthy young adults, and determine if there are significant differences between the exercises for each segment. Method: With fine-wire EMG electrodes inserted into the anterior, middle, and posterior segments of the GMed muscle, 10 healthy young adults performed 6 common, lower-limb rehabilitation exercises. Main Outcome Measures: Recorded EMG activity was normalized, then reported and compared with median activity for each of the GMed segments across the 6 exercises. Results: For the anterior GMed segment, high activity was recorded for the single-leg squat (48\% maximum voluntary isometric contraction [MVIC]), the single-leg bridge (44\% MVIC), and the resisted hip abduction-extension exercise (41\% MVIC). No exercises recorded high activity for the middle GMed segment, but for the posterior GMed segment very high activity was recorded by the resisted hip abduction-extension exercise (69\% MVIC), and high activity was generated by the single-leg squat (48\% MVIC) and side-lie hip abduction (43\% MVIC). For each of the GMed segments, there were significant differences $(P<.05)$ in the median EMG activity levels between some of the exercises and the side-lie clam with large effect sizes favoring these exercises over the side-lie clam. Conclusions: Open-chain hip abduction and single-limb support exercises appear to be effective options for recruiting the individual GMed segments with selection dependent on individual requirements. However, the side-lie clam does not appear to be effective at recruiting the GMed segments, particularly the anterior and middle segments.
\end{abstract}

Keywords: hip, exercise therapy, gluteal muscles, EMG

Gluteus medius (GMed) activity levels have been evaluated across a range of therapeutic exercises. ${ }^{1}$ In most cases, single-leg weight-bearing exercises show greater activity levels than nonweight-bearing exercises when measured with a single-surface electrode over the middle GMed region. ${ }^{1}$ However, the GMed is structurally and functionally composed of 3 unique segments, ${ }^{2}$ and a large proportion of the anterior and posterior segments are deep to the superficially located tensor fascia lata and gluteus maximus, respectively. ${ }^{3}$ The aim of this study was to determine activity levels using fine-wire electromyography (EMG) for the anterior, middle, and posterior GMed segments during 6 common rehabilitation exercises. This may assist clinicians with prescribing targeted rehabilitation programs to prevent, manage, or treat segmental GMed dysfunction that is evident in pathology.,5

\section{Methods}

\section{Participants and Design}

This study was conducted on the same participants as described in a previous publication on gluteus minimus muscle activity. ${ }^{6}$ Ten healthy, active university students (6 males and 4 females) with a mean (SD) age, height, and weight of 23.8 (1.6) years, 177.5 (10) $\mathrm{cm}$, and $79.9(18.5) \mathrm{kg}$, respectively, were recruited for this single-session cross-sectional study. Institutional review board

Moore, Pizzari, McClelland, and Semciw are with the College of Science, Health and Engineering, La Trobe University, Melbourne, VIC, Australia. Moore (damkmoore@hotmail.com) is corresponding author. approval was granted in the spirit of the Declaration of Helsinki (Latrobe University human ethics committee approval, UHEC 13-005).

\section{Instrumentation and Electrode Insertions}

Fine-wire EMG electrodes were inserted into anterior, middle, and posterior segments of GMed as described previously. ${ }^{7,8}$ The electrodes for each GMed segment were connected to a wireless EMG system (Delsys ${ }^{\circledR}$ Inc, Boston, MA). An accelerometer (Trigno; Delsys ${ }^{\circledR}$ Inc) was secured to the top of the iliac crest, distal lateral femur, and distal, anteromedial tibia along with retroreflective markers (Vicon ${ }^{\circledR}$, Oxford, UK) attached to selective anatomical landmarks for the purposes of delineating between exercise repetitions.

\section{Experimental Protocol}

The experimental protocol has been described in detail previously. ${ }^{6}$ Each participant undertook at least 5 minutes of warm-up before performing 6 rehabilitation exercises in a randomized order paced to a metronome (Supplementary Video 1 [available online]). Three trials of 6 repetitions were performed for the single-leg squat (40 bpm), the single-leg bridge (40 bpm), the side-lie hip abduction (50 bpm), the side-lie clam (40 bpm), the running man exercise (90 bpm), and the resisted hip abduction-extension exercise $(60 \mathrm{bpm})$ with 2 minutes of rest between the trials and exercises. A series of maximum voluntary isometric contractions (MVICs) (across 6 hip actions) were performed for data normalization (Supplementary Table S1 [available online]). 


\section{Statistical Analysis}

The R statistical software package (version 3.4.1; https://cran.rproject.org/) was used for analysis. The EMG data processing has been described in detail previously. ${ }^{6}$ Muscle activity was described qualitatively for each exercise using the following criteria: low (0\%-20\% MVIC), moderate (21\%-40\% MVIC), high (41\%-60\% MVIC), and very high ( $>60 \%$ MVIC). To determine if normalized (\%MVIC) muscle activity for each segment differed across exercises, a nonparametric Friedman test was used along with Nemenyi post hoc tests $(P<.05)$. An effect size was calculated by dividing the chi-square $\left(\chi^{2}\right)$ test with the square root of the sample size.

\section{Results}

\section{Participants}

Three participants' data were excluded for each GMed segment due to artifact.

\section{GMed Anterior Median Activity}

High median (interquartile range) activity was recorded for the single-leg squat (48\% [11]\% MVIC), the single-leg bridge (44\% [9]\% MVIC), and the resisted hip abduction-extension exercise (41\% [8]\% MVIC) (Figure 1A). Moderate activity was generated by the side-lie hip abduction (36\% [17]\% MVIC) and the running man exercise (35\% [12]\% MVIC). Low activity (0\%-20\% MVIC) was recorded during side-lie clam (1\% [1]\% MVIC).

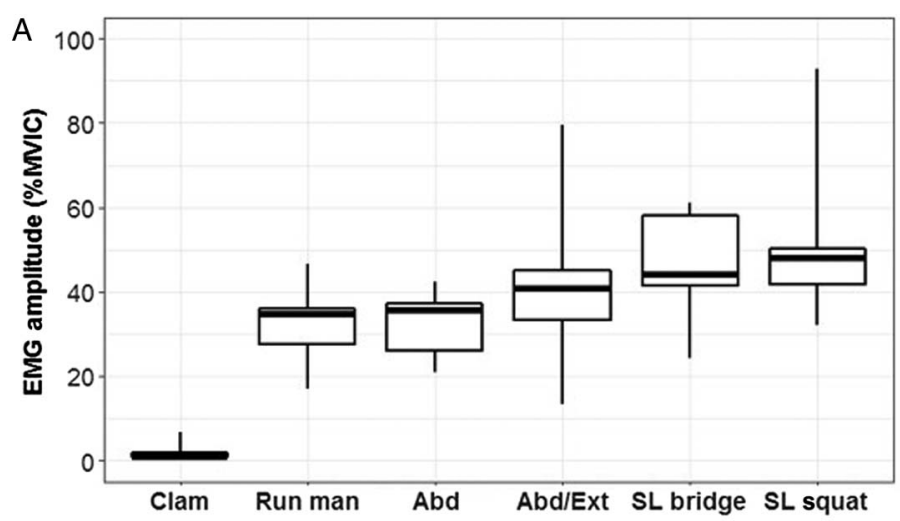

B

\begin{tabular}{|c|c|c|c|c|c|c|}
\hline & Clam & Run man & Abd & Abd/Ext & SL bridge & SL squat \\
\hline Clam & & $\begin{array}{l}2.63 \\
(.429)\end{array}$ & $\begin{array}{l}2.83 \\
(.342)\end{array}$ & $\begin{array}{l}4.44 \\
(.021)\end{array}$ & $\begin{array}{l}5.25 \\
(.003)\end{array}$ & $\begin{array}{l}6.06 \\
(.000)\end{array}$ \\
\hline Run man & & & $\begin{array}{l}0.20 \\
(.000)\end{array}$ & $\begin{array}{l}1.82 \\
(.793)\end{array}$ & $\begin{array}{l}2.63 \\
(.429)\end{array}$ & $\begin{array}{l}3.43 \\
(.146)\end{array}$ \\
\hline Abd & & & & $\begin{array}{l}1.62 \\
(.863)\end{array}$ & $\begin{array}{l}2.42 \\
(.522)\end{array}$ & $\begin{array}{l}3.23 \\
(.200)\end{array}$ \\
\hline Abd/Ext & & & & & $\begin{array}{l}0.81 \\
(.993)\end{array}$ & $\begin{array}{l}1.62 \\
(.863)\end{array}$ \\
\hline SL bridge & & & & & & $\begin{array}{l}0.81 \\
(.993)\end{array}$ \\
\hline
\end{tabular}

Figure 1 - (A) Box plots illustrating median, interquartile range, and range of GMed anterior activity levels across the 6 exercises. (B) Post hoc pairwise comparison between exercises with ES for GMed anterior median activity. Abd/Ext indicates abduction/extension; EMG, electromyography; ES, effect size; GMed, gluteus medius; MVIC, maximum voluntary isometric contraction; SL, side lie.
There were significant within-participant effects across all the exercises for GMed anterior median activity $\left(\chi^{2}{ }_{5}=23.98\right.$, $P \leq .001)$.

The side-lie clam had significantly lower activity levels than the single-leg bridge, the single-leg squat, and the resisted hip abduction-extension exercise with large effect sizes generated (Figure 1B).

\section{GMed Middle Median Activity}

Moderate median (interquartile range) activity was generated by the single-leg squat (40\% [9]\% MVIC), the side-lie hip abduction (37\% [16]\% MVIC), the single-leg bridge (36\% [21]\% MVIC), the resisted hip abduction-extension exercise (33\% [26]\% MVIC), and the running man exercise (33\% [20]\% MVIC) (Figure 2A). Low activity was recorded by the side-lie clam (4\% [3]\% MVIC).

There were significant within-participant effects across all the exercises for GMed middle median activity $\left(\chi^{2}{ }_{5}=18.76, P \leq .002\right)$.

The side-lie clam had significantly lower activity levels than the single-leg bridge, the single-leg squat, and the side-lie hip abduction with large effect sizes generated (Figure 2B).

\section{GMed Posterior Median Activity}

Very high median (interquartile range) activity was recorded by the resisted hip abduction-extension exercise (69\% [47]\% MVIC) (Figure 3A). High activity was generated by the single-leg squat (48\% [24]\% MVIC) and the side-lie hip abduction (43\% [23]\% MVIC). Moderate activity was elicited by the single-leg bridge (39\% [11]\% MVIC) and the running man exercise (33\% [47]\%

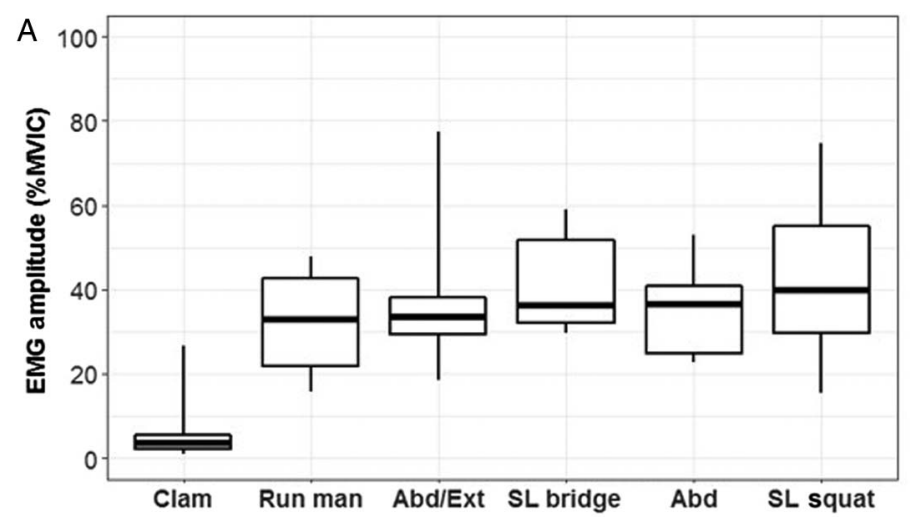

B

\begin{tabular}{|c|c|c|c|c|c|c|}
\hline & Clam & \begin{tabular}{|l|} 
Run man \\
\end{tabular} & $\mathrm{Abd} /$ Ext & SL bridge & Abd & SL squat \\
\hline Clam & & $\begin{array}{l}1.22 \\
(.200)\end{array}$ & $\begin{array}{l}1.30 \\
(.146)\end{array}$ & $\begin{array}{l}1.99 \\
(.003)\end{array}$ & $\begin{array}{l}1.53 \\
(.049)\end{array}$ & $\begin{array}{l}1.99 \\
(.003)\end{array}$ \\
\hline Run man & & & $\begin{array}{l}0.08 \\
(.000)\end{array}$ & $\begin{array}{l}0.76 \\
(.710)\end{array}$ & $\begin{array}{l}0.31 \\
(.993)\end{array}$ & $\begin{array}{l}0.76 \\
(.710)\end{array}$ \\
\hline Abd/Ext & & & & $\begin{array}{l}0.69 \\
(.793)\end{array}$ & $\begin{array}{l}0.23 \\
(.998)\end{array}$ & $\begin{array}{l}0.69 \\
(.793) \\
\end{array}$ \\
\hline SL bridge & & & & & $\begin{array}{l}0.46 \\
(.956)\end{array}$ & $\begin{array}{l}0.00 \\
(.000) \\
\end{array}$ \\
\hline Abd & & & & & & \begin{tabular}{|l}
0.46 \\
$(.956)$
\end{tabular} \\
\hline
\end{tabular}

Figure 2 - (A) Box plots illustrating median, interquartile range, and range of GMed middle activity levels across the 6 exercises. (B) Post hoc pairwise comparison between exercises with ES for GMed middle median activity. Abd/Ext indicates abduction/extension; EMG, electromyography; ES, effect size; GMed, gluteus medius; MVIC, maximum voluntary isometric contraction; SL, side lie. 


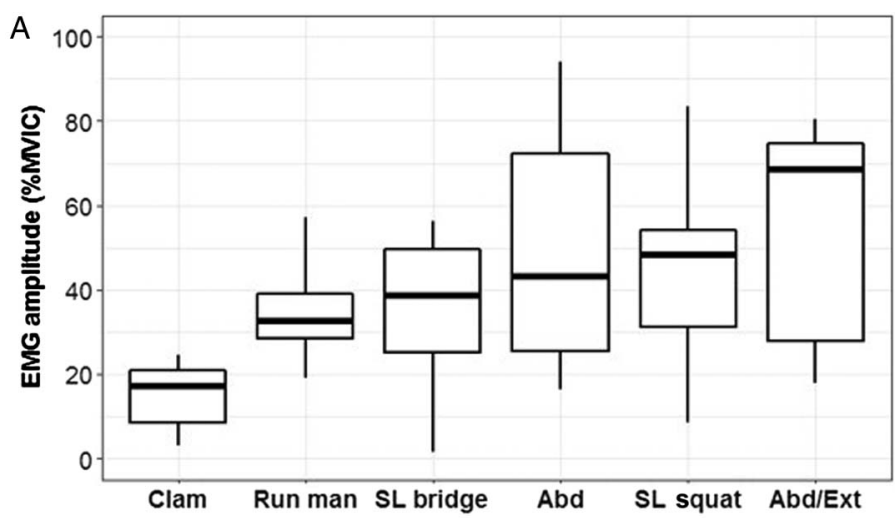

B

\begin{tabular}{|c|c|c|c|c|c|c|}
\hline & Clam & Run man & SL bridge & Abd & SL squat & $\mathrm{Abd} / \mathrm{Ext}$ \\
\hline Clam & & $\begin{array}{l}.23 \\
(.200)\end{array}$ & $\begin{array}{l}2.22 \\
(.618)\end{array}$ & $\begin{array}{l}4.65 \\
(.013) \\
\end{array}$ & $\begin{array}{l}.43 \\
(.146)\end{array}$ & \begin{tabular}{|l|}
4.04 \\
$(.049)$ \\
\end{tabular} \\
\hline Run man & & & $\begin{array}{l}1.01 \\
(.980)\end{array}$ & $\begin{array}{l}1.41 \\
(.918)\end{array}$ & $\begin{array}{l}0.20 \\
(1.000)\end{array}$ & $\begin{array}{l}0.81 \\
(.993) \\
\end{array}$ \\
\hline SL bridge & & & & $\begin{array}{l}2.42 \\
(.522)\end{array}$ & $\begin{array}{l}1.21 \\
(.956)\end{array}$ & \begin{tabular}{|l|}
1.82 \\
$(.793)$
\end{tabular} \\
\hline Abd & & & & & $\begin{array}{l}1.21 \\
(.956)\end{array}$ & \begin{tabular}{|l|}
0.61 \\
$(.998)$ \\
\end{tabular} \\
\hline SL squat & & & & & & $\begin{array}{l}0.61 \\
(.998)\end{array}$ \\
\hline
\end{tabular}

Figure 3 - (A) Box plots illustrating median, interquartile range, and range of GMed posterior activity levels across the 6 exercises. (B) Post hoc pairwise comparison between exercises with ES for GMed middle posterior activity. Abd/Ext indicates abduction/extension; EMG, electromyography; ES, effect size; GMed, gluteus medius; MVIC, maximum voluntary isometric contraction; SL, side lie.

MVIC). Low activity was recorded by the side-lie clam (17\% [12]\% MVIC).

There were significant within-participant effects across all the exercises for GMed posterior median activity $\left(\chi^{2}{ }_{5}=13.61\right.$, $P \leq .02)$.

The side-lie clam had significantly lower activity levels than the side-lie hip abduction and the resisted hip extension-abduction exercise with large effect sizes generated (Figure 3B).

\section{Discussion}

This study investigated activity levels of anterior, middle, and posterior segments of the GMed during common rehabilitation exercises in healthy young adults. These results indicate that simple rehabilitation exercises with minimal equipment can be prescribed to optimally target the individual GMed segments for strengthening (>40\% MVIC). ${ }^{9}$ The single-leg squat generated relatively high activity levels in all 3 GMed segments. The resisted hip abductionextension exercise generated high to very high activity in the anterior and posterior segments. The single-leg bridge generated high activity in the anterior segment, whereas the side-lie hip abduction recorded high activity in the posterior segment. The side-lie clam, in contrast, generated low activity in each of the GMed segments.

The single-leg squat challenges all 3 GMed segments to maintain pelvic equilibrium while controlling hip adduction and internal rotation, as the body's center of mass is lowered toward the ground. ${ }^{10}$ This exercise should be considered a valuable functional exercise for strengthening all 3 GMed segments due to the high activity recorded in each segment. This result compares well with a previous review ${ }^{1}$ that found high to very high GMed activity levels were recorded for this exercise.

The running man exercise and the resisted hip abductionextension exercise are performed in single-limb standing, requiring sizeable hip abduction torques from all 3 segments to maintain pelvic equilibrium. ${ }^{10}$ This is reflected by the moderate to very high activity levels recorded across the GMed segments and would be potentially effective for targeted segmental strengthening, where at least high activity was generated. The results of this study compare favorably with a recent study on healthy older women that investigated 3 different stance-leg, hip-hitch exercises with high to very high activity levels generated for all 3 GMed segments. ${ }^{5}$

The single-leg bridge is a popular rehabilitation exercise that has been reported to generate high GMed activity. ${ }^{1}$ With a substantial external torque created from the unsupported leg, moderate to high activity levels in all 3 GMed segments were required presumably to contribute to a hip extension and internal rotation torque for maintaining a neutral pelvic position throughout the exercise. ${ }^{10}$

Both the side-lie abduction and the side-lie clam are commonly prescribed rehabilitation exercises that have been previously reported to generate moderate to very high GMed activity. ${ }^{1}$ The side-lie abduction provides the clinician with a reasonably effective open chain exercise for targeting the individual segments with moderate to high activity levels generated in each of the segments.

By contrast, the side-lie clam was demonstrated in this study to be an ineffective exercise for targeting all 3 segments, with a smaller antigravity lever arm to overcome. Morphologically, the anterior and middle segments do not have favorable moment arms in the transverse plane for external rotation compared with the posterior segment, ${ }^{10}$ and the results from this study compare well with a recent study ${ }^{5}$ on healthy older women performing the sidelie clam that also showed low activity levels in all 3 segments.

Based on the results of this study, open-chain hip abduction and single-limb support exercises appear to be effective options for strengthening all GMed segments to potentially counteract dysfunction with selection based on the individual's functional requirements. The side-lie clam, in comparison, does not appear to be particularly effective at GMed recruitment, especially the anterior and middle segments.

\section{Limitations}

The sample size in this study was limited due to the invasive nature of the procedure. Despite this, significant differences in activation levels were still observed, and the use of effect sizes has provided an estimate of the magnitude of difference between exercises. For pragmatic reasons, the rehabilitation exercises included in this study are commonly prescribed in the clinic with the assumption as for any exercise that adding external load will have an effect on recorded exercise intensity level. Caution also needs to be applied when generalizing these results to clinical populations such as those with hip osteoarthritis and lateral hip pain.

\section{Acknowledgments}

This study was supported by an Australian Government Research Training Scholarship. The authors have no conflicts of interest to disclose. 


\section{References}

1. Ebert JR, Edwards PK, Fick DP, Janes GC. A systematic review of rehabilitation exercises to progressively load the gluteus medius. J Sport Rehabil. 2017;26(5):418-436. PubMed ID: 27632888 doi:10.1123/jsr.2016-0088

2. Flack NA, Nicholson HD, Woodley SJ. The anatomy of the hip abductor muscles. Clin Anat. 2014;27(2):241-253. PubMed ID: 23625344 doi:10.1002/ca.22248

3. Semciw AI, Neate R, Pizzari T. A comparison of surface and fine wire EMG recordings of gluteus medius during selected maximum isometric voluntary contractions of the hip. J Electromyogr Kinesiol. 2014;24(6): 835-840. PubMed ID: 25245248 doi:10.1016/j.jelekin.2014.08.015

4. Zacharias A, Pizzari T, English DJ, Kapakoulakis T, Green RA. Hip abductor muscle volume in hip osteoarthritis and matched controls. Osteoarthritis Cartilage. 2016;24(10):1727-1735. PubMed ID: 27163446 doi:10.1016/j.joca.2016.05.002

5. Ganderton C, Pizzari T, Cook J, Semciw A. Gluteus minimus and gluteus medius muscle activity during common rehabilitation exercises in healthy postmenopausal women. J Orthop Sports Phys Ther. 2017;47(12):914-922. PubMed ID: 29034801 doi:10.2519/jospt. 2017.7229
6. Moore D, Semciw AI, McClelland J, Wajswelner H, Pizzari T. Rehabilitation exercises for the gluteus minimus muscle segmentsan electromyography study. J Sport Rehabil. 2019;1-8. doi:10. 1123/jsr.2017-0262

7. Semciw AI, Pizzari T, Green RA. Technical application and the level of discomfort associated with an intramuscular electromyographic investigation into gluteus minimus and gluteus medius. Gait Posture. 2013;38(1):157-160. PubMed ID: 23127355 doi:10.1016/j.gaitpost. 2012.10.010

8. Semciw AI, Green RA, Pizzari T, Briggs C. Verification of a standardized method for inserting intramuscular EMG electrodes into uniquely oriented segments of gluteus minimus and gluteus medius. Clin Anat. 2013;26(2):244-252. PubMed ID: 22374796 doi:10.1002/ca.22055

9. Andersen LL, Magnusson SP, Nielsen M, Haleem J, Poulsen K, Aagaard P. Neuromuscular activation in conventional therapeutic exercises and heavy resistance exercises: implications for rehabilitation. Phys Ther. 2006;86(5):683-697. PubMed ID: 16649892

10. Neumann DA. Kinesiology of the hip: a focus on muscular actions. J Orthop Sports Phys Ther. 2010;40(2):82-94. PubMed ID: 20118525 doi:10.2519/jospt.2010.3025 